\title{
Source amplitudes for active exterior cloaking
}

\author{
A. N. Norris ${ }^{a}$, F. A. Amirkulova ${ }^{a}$, W. J. Parnell ${ }^{b}$ \\ (a) Mechanical and Aerospace Engineering, Rutgers University, \\ Piscataway NJ 08854-8058, USA \\ (b) School of Mathematics, Alan Turing Building, \\ University of Manchester, Oxford Road, \\ Manchester, M13 9PL, UK
}

October 13, 2018

\begin{abstract}
The active cloak comprises a discrete set of multipole sources that destructively interfere with an incident time harmonic scalar wave to produce zero total field over a finite spatial region. For a given number of sources and their positions in two dimensions it is shown that the multipole amplitudes can be expressed as infinite sums of the coefficients of the incident wave decomposed into regular Bessel functions. The field generated by the active sources vanishes in the infinite region exterior to a set of circles defined by the relative positions of the sources. The results provide a direct solution to the inverse problem of determining the source amplitudes. They also define a broad class of non-radiating discrete sources.
\end{abstract}

\section{Introduction}

Cloaking is intended to make an object undetectable to incident waves. The approaches proposed consist mainly of two quite distinct types of cloaking, namely passive and active. Passive cloaking requires devising a metamaterial that can steer the wave energy around the object regardless of the incident wave. Our interest here is with active cloaking, specifically in situations where the active sources lie in the exterior of the region containing the cloaked object. We call this configuration active exterior cloaking in keeping with prior terminology [1.

Despite the dominant interest in passive cloaking devices, active exterior cloaking has been investigated quite extensively [2, 1, 3, 4, 5]. Miller [2] proposed creating a cloaking region by measuring particle motion near the surface of the cloaking zone while simultaneously exciting appropriate surface sources where each source amplitude depends on the measurements at all sensing points. As an active cloaking method this approach is limited because it does not provide a unique relationship between the incident field on the one hand, and the source amplitudes on the other. A solution to this problem was provided by Vasquez et al. [1, 3] in the context of active exterior cloaking for the 2D Helmholtz equation. They 
used Green's formula and addition theorems for Bessel functions to formulate an integral equation, which was converted to a linear system of equations for the unknown amplitudes. Crucially, the integral equation provides the source amplitudes as linear functions of the incident wave field. Vasquez et al. also showed, by construction, that active cloaking can be realized using as few as three active sources in 2D. A more explicit form of the linear relation for the source amplitudes as a function of the incident field was developed in [5]. Multipolar sources were used to reproduce Miller's cloak [2], and numerical results were compared with SVD solutions of the linearized system [1, 3]. The approach of [1, 3] was generalized in [4] to handle the 3D Helmholtz equation, seeking non resonant frequencies of the cloaked object. Further analysis and extension of the methods to the quasistatic regime relevant to Laplace's equation can be found in [6, 7, 8]. The active source method of Vasquez et al. has also been adapted to create illusion effects so that an object outside the cloaking region can be made to appear like another object [9].

In this paper we demonstrate that the integral representation of Vasquez et al. 55 for the source amplitudes can be reduced to closed-form explicit formulas. This obviates the need to reduce the integral equation of Vasquez et al. [1, 3] to a system of linear equations, which must then be solved numerically, or to evaluate line integrals, as proposed in [5]. We provide analytical expressions for the source amplitude coefficients for general incidence as well as plane wave incidence. The expressions involve no more than sums of cylinder functions which can be truncated to achieve any desired accuracy. We also prove that the field generated by the active sources vanishes in the infinite region exterior to a set of circles defined by the relative positions of the sources. The active source field, by construction, cancels the incident field in the cloaked region, which is defined by the region interior to the same circular areas. The analytical results are verified by calculation of the farfield and the nearfield amplitudes, which are shown to vanish when the summation is accurately evaluated.

The non-radiating nature of the active field has relevance to the inverse source problem [10]. Although for this problem, some uniqueness results are available for restricted forms of sources, e.g. "minimum energy sources" [11], in general the solution to this problem is know to be non-unique [12]. Here we develop the solution of the active cloaking problem as a new family of non-radiating sources, with the property that they cancel a given incident field over a finite region.

We begin in 92 with a statement of the problem, a review of the governing equations, and a summary of the main results, given in eqs. (2) and (7). The basic integral relation of Vasquez et al. [5] is derived in \$3, from which the main results are shown to follow. Some example applications of the new formulas are presented in \$4. Some implications of the general results are discussed in $\$ 5$ and conclusions are given in $\$ 6$.

\section{The problem and its solution}

\subsection{Problem overview}

The active cloaking devices considered here operate in two dimensions, and consist of arrays of point multipole sources located at positions $\mathbf{x}_{m} \in \mathbb{R}^{2}, m=\overline{1, M}$, see Figure 1 . The active sources lie in the exterior region with respect to the cloaked region $C$ and this type of cloaking may therefore be called "active exterior cloaking" [1. Objects are undetectable in the cloaked region by virtue of the destructive interference of the sources and the incident field with the result that the total wave amplitude vanishes in the cloaked region $C$. The advantages of this type of cloaking device are: (i) the cloaked region is not completely surrounded by a single cloaking device; (ii) only a small number of active sources are needed; (iii) the procedure 


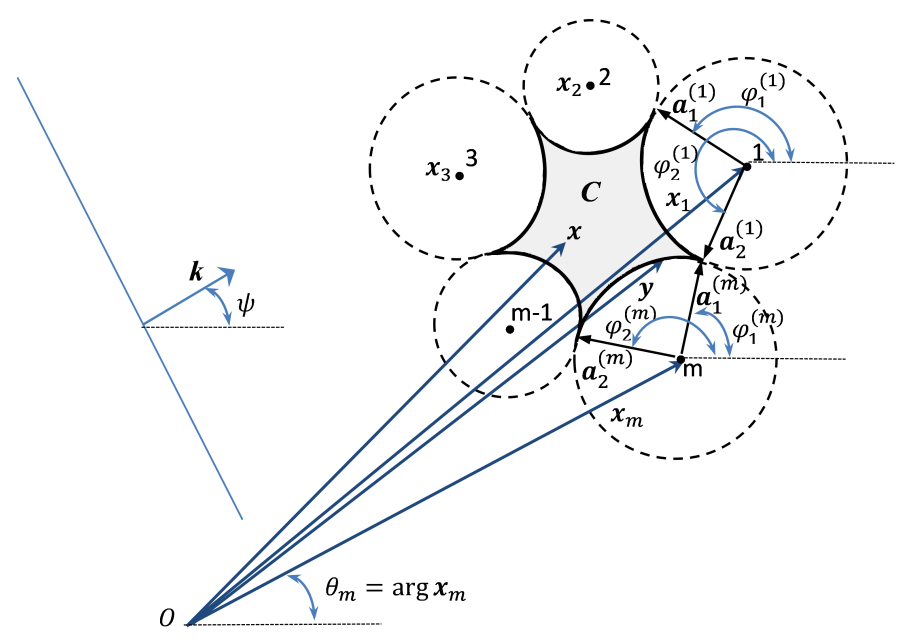

Figure 1: Insonification of the actively cloaked region $C$ generated by $M$ active point multipole sources at $\mathbf{x}_{m}, m=\overline{1, M}$. The region $R$ is defined as the interior of the union of the dashed circular arcs, that is, the combined area comprising $C$ and the $M$ circular domains. The incident field in this case is a plane wave with wave vector $\mathbf{k}$ in the direction $\psi$.

works for broadband input sources. A disadvantage of the active cloaking approach is that the fields near the ideal sources may become uncontrollably large. Realistically these would be replaced by regions of finite extent and thus their magnitude is reduced. A further disadvantage of the method is that the incident field must be known. However we note that with the approach proposed in this paper, the new expressions require only the expansion of the incident field into entire cylindrical waves, as compared with the line integrals derived in [4] which require knowledge of the incident field and its normal derivative.

The shaded region in Figure 1 denotes the cloaked zone $C$ generated by $M$ active point multipole sources. The boundary of $C$ is the closed concave union of the circular arcs $m=$ $\overline{1, M},\left\{a_{m}, \phi_{1}^{(m)}, \phi_{2}^{(m)}\right\}$ associated with the source at $\mathbf{x}_{m}$. In the general case $\left\{a_{m}, \phi_{1}^{(m)}, \phi_{2}^{(m)}\right\}$ are distinct for different values of $m$. Note that the wave incidence shown in Figure 1 is a plane wave although the solution derived below is for arbitrary incidence. The inverse problem to be solved is to find the amplitudes of the active sources as a function of the incident wave, and to prove that the cloaked region is indeed the closed region $C$.

\subsection{Basic equations}

We assume time harmonic dependence $e^{-i \omega t}$ which is omitted hereafter and consider the scalar Helmholtz equation in two dimensions. Thus the method proposed here is applicable to any physical situation described as such. For ease of discussion however let us consider the case of acoustics, so that the governing equation for the (time harmonic) pressure $u(\mathbf{x})$ is

$$
\nabla^{2} u+k^{2} u=s
$$

where $k=\omega / c$ is the wavenumber, $c$ the acoustic speed, and the term $s$ represents sources. For a given incident wave we assume there is an additional field resulting from the active 
sources which exactly cancels the incident wave in some bounded region $C$. This additional wave field is caused by the $M$ multipole sources located at $\mathbf{x}_{m}, m=\overline{1, M}$. The assumed form of the total field $u$, the incident wave $u_{i}$, and the active source field $u_{d}$ are, respectively

$$
\begin{aligned}
u & =u_{i}+u_{d}, \\
u_{i} & =\sum_{n=-\infty}^{\infty} A_{n} U_{n}^{+}(\mathbf{x}), \\
u_{d} & =\sum_{m=1}^{M} \sum_{n=-\infty}^{\infty} b_{m, n} V_{n}^{+}\left(\mathbf{x}-\mathbf{x}_{m}\right),
\end{aligned}
$$

where the wave functions $U_{n}^{ \pm}(\mathbf{x})$ and $V_{n}^{ \pm}(\mathbf{x})$ are defined by

$$
U_{n}^{ \pm}(\mathbf{x})=J_{n}(k|\mathbf{x}|) e^{ \pm i n \arg \mathbf{x}}, \quad V_{n}^{ \pm}(\mathbf{x})=H_{n}^{(1)}(k|\mathbf{x}|) e^{ \pm i n \arg \mathbf{x}} .
$$

Here $\arg \mathbf{x} \in[0,2 \pi)$ and $\arg (-\mathbf{x})=\arg \mathbf{x} \pm \pi \in[0,2 \pi)$. Define the derivative functions $U_{n}^{ \pm \prime}(\mathbf{a})$ as

$$
U_{n}^{ \pm \prime}(\mathbf{a})=J_{n}^{\prime}(k a) e^{ \pm i n \arg \mathbf{a}} .
$$

In the following we write $U_{0}$ and $V_{0}$, with obvious meaning. Note that the functions $U_{n}^{ \pm}(\mathbf{x})$ and $V_{n}^{ \pm}(\mathbf{x})$ possess the properties

$$
U_{n}^{ \pm}(-\mathbf{x})=(-1)^{n} U_{n}^{ \pm}(\mathbf{x}), \quad V_{n}^{ \pm}(-\mathbf{x})=(-1)^{n} V_{n}^{ \pm}(\mathbf{x}) .
$$

The active source field $u_{d}$ in (2C) is of the same form as considered by Vasquez et al. [3, eq. (5)]. The three dimensional analog is given in [4, eq. (40)]. The coefficients $A_{n}$, which define the incident field, include as a special case plane wave incidence in the direction $\psi$ $\left(A_{n}=i^{n} e^{-i n \psi}\right)$.

The active cloaking problem is now to find (i) the coefficients $b_{m, n}$ such that the total field $u$ vanishes inside some compact region $C$, and (ii) to define the region $C$.

\subsection{Summary of main results}

The principal results can be summarized in two theorems. The first provides necessary and sufficient conditions on the source amplitudes $b_{m, n}$ in order to ensure cloaking in the region $C$ and a non-radiating source field $u_{d}$. The second provides the explicit expressions for the active source amplitudes.

Theorem 1. Necessary and sufficient conditions on the active source coefficients $b_{m, l}$ in order to ensure zero total field $\left(u_{i}+u_{d}=0\right)$ inside $C$ and no radiated field $\left(u_{d} \rightarrow 0\right.$ in the far field) are

$$
\forall n \in \mathbb{Z}: \quad \sum_{m=1}^{M} \sum_{l=-\infty}^{\infty} b_{m, l} \times\left\{\begin{array}{l}
U_{n-l}^{-}\left(\mathbf{x}_{m}\right)=0, \\
V_{n-l}^{-}\left(\mathbf{x}_{m}\right)=-A_{n} .
\end{array}\right.
$$

These identities provide a useful means to quantify error in active cloaking as will be seen later on. We now state the explicit form for the source amplitudes, together with the shape of the cloaked region $C$ and the region in which the source field vanishes. 
Theorem 2. Given $M$ active sources located at $\mathbf{x}_{m}, m=\overline{1, M}$, the required active source amplitude coefficients for the general incidence (2b) are

$$
\begin{aligned}
& b_{m, l}= \sum_{n=-\infty}^{\infty} b_{m, l n} A_{n} \quad \text { where } \\
& b_{m, l n}=\frac{k a_{m}}{4} \sum_{p=-\infty}^{\infty} U_{n+p}^{+}\left(\mathbf{x}_{m}\right) \frac{(-1)^{p}}{l+p}\left[J_{p}\left(k a_{m}\right) J_{l}^{\prime}\left(k a_{m}\right)-J_{p}^{\prime}\left(k a_{m}\right) J_{l}\left(k a_{m}\right)\right] \\
& \quad \times\left[e^{-i(l+p) \phi_{2}^{(m)}}-e^{-i(l+p) \phi_{1}^{(m)}}\right] .
\end{aligned}
$$

This ensures cloaking (zero total field) in the region $C$ which is the closed and bounded domain formed by taking its boundary as the closed concave union of the circular arcs defined by $\left\{a_{m}, \phi_{1}^{(m)}, \phi_{2}^{(m)}\right\}$ and denoted as $\partial C_{m}$, see Figure 1. These coefficients also ensure that the radiated field from $u_{d}$ is identically zero in the region exterior to all of the circles centered at the source points:

$$
u_{d}(\mathbf{x})=0 \text { for } \mathbf{x} \in \mathbb{R}^{2} / R, \quad R \equiv C \bigcup\left\{\mathbf{x}:\left|\mathbf{x}-\mathbf{x}_{m}\right| \leq a_{m}, m=\overline{1, M}\right\} .
$$

This is the exterior to the union of the dashed circular arcs in Figure 1.

An alternative and more concise formulation of eq. (7b) is obtained using the notation of eq. (3) with $\mathbf{a}_{i}^{(m)} \equiv a_{m} \hat{\mathbf{e}}\left(\phi_{i}^{m}\right),(i=1,2)$,

$$
b_{m, l n}=\left.\frac{1}{4} k a_{m} \sum_{p=-\infty}^{\infty} U_{n+p}^{+}\left(\mathbf{x}_{m}\right) \frac{(-1)^{p}}{l+p}\left[U_{p}^{-}(\mathbf{a}) U_{l}^{-\prime}(\mathbf{a})-U_{p}^{-\prime}(\mathbf{a}) U_{l}^{-}(\mathbf{a})\right]\right|_{\mathbf{a}_{1}^{(m)}} ^{\mathbf{a}_{2}^{(m)}}
$$

where $\hat{\mathbf{e}}\left(\phi_{i}^{m}\right)$ is a unit vector subtended at angle $\phi_{i}^{m}$, as illustrated in Figure 1.

An important case for which the summation in (7a) can be simplified is plane wave incidence. Assuming the incident field is a unit amplitude plane wave in direction $\psi, u_{i}=u_{\psi}$ defined by $A_{n}=i^{n} e^{-i n \psi}$, results in

$$
b_{m, l}=\left.u_{\psi}\left(\mathbf{x}_{m}\right) \frac{k a_{m}}{4} \sum_{p=-\infty}^{\infty} \frac{i^{p} e^{i p \psi}}{l+p}\left[U_{p}^{-}(\mathbf{a}) U_{l}^{-{ }^{\prime}}(\mathbf{a})-U_{p}^{-\prime}(\mathbf{a}) U_{l}^{-}(\mathbf{a})\right]\right|_{\mathbf{a}_{1}^{(m)}} ^{\mathbf{a}_{2}^{(m)}}, \quad \begin{gathered}
\text { plane wave } \\
\text { incidence. }
\end{gathered}
$$

The form of the coefficients $b_{m, l}$ is discussed further below. Note that the term in (7b), (9) and in (10), corresponding to $p+l=0$ is zero, which follows from l'Hôpital's rule, or otherwise.

Theorems 1 and 2 are proved in the next section.

\section{Proofs of Theorems 1 and 2}

\subsection{Theorem 1: Necessary and sufficient conditions on the source amplitudes}

We first prove the constraints on the source coefficients $b_{m, l}$ given by Theorem 1, and at the same time show that they may be interpreted in terms of the near- and far-field of the 
active sources. To this end, we express $u_{d}$ in two different forms using the generalized Graf addition theorem [13, eq. (9.1.79)],

$$
V_{l}^{+}(\mathbf{x}-\mathbf{y})=\sum_{n=-\infty}^{\infty} \begin{cases}V_{n}^{+}(\mathbf{x}) U_{n-l}^{-}(\mathbf{y}), & |\mathbf{x}|>|\mathbf{y}| \\ U_{n}^{+}(\mathbf{x}) V_{n-l}^{-}(\mathbf{y}), & |\mathbf{x}|<|\mathbf{y}| .\end{cases}
$$

Let us first consider the radiated field, assuming that $u_{d}$ does not radiate energy into the far field. The first of (11), for $|\mathbf{x}|>|\mathbf{y}|$, allows us to rewrite $u_{d}$ as a sum of multipoles at the origin:

$$
u_{d}=\sum_{n=-\infty}^{\infty} F_{n} V_{n}^{+}(\mathbf{x}) \text { for }|\mathbf{x}|>\max \left(\left|\mathbf{x}_{m}\right|+a_{m}\right) \text {, }
$$

where

$$
F_{n}=\sum_{m=1}^{M} \sum_{l=-\infty}^{\infty} b_{m, l} U_{n-l}^{-}\left(\mathbf{x}_{m}\right)
$$

Define the farfield amplitude function $f(\theta), \theta=\arg \hat{\mathbf{x}}$, such that

$$
u_{d}(\mathbf{x})=f(\theta) \frac{e^{i k|\mathbf{x}|}}{(k|\mathbf{x}|)^{1 / 2}}+\mathrm{O}\left((k|\mathbf{x}|)^{-3 / 2}\right), \quad|\mathbf{x}| \rightarrow \infty .
$$

The farfield amplitude function follows from the asymptotic form of the Hankel functions as

$$
f(\theta)=\sum_{n=-\infty}^{\infty} f_{n} e^{i n \theta}, \quad f_{n}=\left(\frac{2}{\pi}\right)^{1 / 2} i^{-\left(n+\frac{1}{2}\right)} F_{n} .
$$

A measure of the nondimensional total power radiated by the sources is given by the nonnegative far-field flux parameter

$$
\sigma_{r}=\int_{0}^{2 \pi} \mathrm{d} \theta|f(\theta)|^{2}=4 \sum_{n=-\infty}^{\infty}\left|F_{n}\right|^{2}
$$

Since $u_{d}$ does not radiate energy into the far field, the active sources must vanish, so that $F_{n}=0 \forall n$. Imposing this in (13) ensures the necessity of $(6)_{1}$. The sufficiency of $(6)_{1}$ is seen immediately by substituting (6) 1 into (13) and (12) which gives $u_{d}=0$.

Now let us consider the near-field inside the cloaked region $C$ where we assume that the cloaked region contains the origin and the total field is zero inside $C$, i.e. $u_{i}+u_{d}=0$. Using the second identity in (11), the active source field $u_{d}$ can be expressed in a form that is valid in the neighborhood of the origin (assuming $\left|\mathbf{x}_{m}\right|>a_{m} \forall m$ ),

$$
u_{d}=\sum_{n=-\infty}^{\infty} E_{n} U_{n}^{+}(\mathbf{x}) \text { for }|\mathbf{x}|<\min \left(\left|\mathbf{x}_{m}\right|-a_{m}\right) \text {, }
$$

where

$$
E_{n}=\sum_{m=1}^{M} \sum_{l=-\infty}^{\infty} b_{m, l} V_{n-l}^{-}\left(\mathbf{x}_{m}\right)
$$


The total field vanishing in some neighbourhood of the origin thus implies that $E_{n}+A_{n}$ vanishes for every value of $n$. This gives rise to the necessary condition (6) $)_{2}$. Sufficiency is once again immediate by assuming the form (6) ${ }_{2}$ and back-substituting into the forms of $u_{d}$ and $u_{i}$ above. 2 .

Further implications of this result are explored after we complete the proof of Theorem

\subsection{Theorem 2: Explicit forms for the active source amplitudes}

The Green's function $g\left(\mathbf{x}, \mathbf{x}^{\prime}\right)$ is defined as the solution of (1) for source $s=\delta\left(\mathbf{x}-\mathbf{x}^{\prime}\right)$, i.e. $g\left(\mathbf{x}, \mathbf{x}^{\prime}\right)=-\frac{i}{4} V_{0}\left(\mathbf{x}-\mathbf{x}^{\prime}\right)$. Consider a region $D$ such as that depicted in Figure 2, chosen so that it does not contain any sources. We will determine the explicit form for the active source amplitudes together with the form of $D$ that ensures cloaking. The latter, already introduced as $C$, is the region depicted in Figure 1.

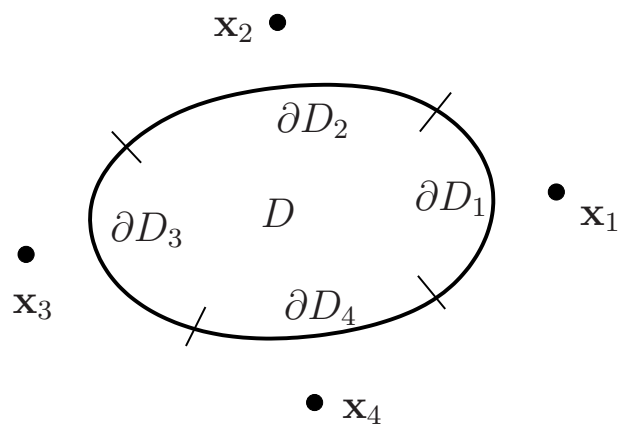

Figure 2: A configuration of $M=4$ sources, and a region $D$ in which the integral identity (19) holds.

By assumption, both $u_{i}$ and $u_{d}$ satisfy the homogeneous Helmholtz equation in $D$ (eq. (1) with $s=0 \forall \mathrm{x} \in D$ ), and therefore

$$
\int_{\partial D} \mathrm{~d} S(\mathbf{y})\left[v(\mathbf{y}) \partial_{n} g(\mathbf{y}, \mathbf{x})-g(\mathbf{y}, \mathbf{x}) \partial_{n} v(\mathbf{y})\right]=v(\mathbf{x}), \quad v=\left\{u_{i}, u_{d}\right\}, \quad \mathbf{x} \in D .
$$

where $\partial D$ is the boundary of $D$ depicted in Figure 2 as the union of the $\operatorname{arcs} \partial D_{m}, m=\overline{1, M}$ and it is traversed counter-clockwise. We wish to determine the cloaked region $C \subset D$ which is defined by its property that the total field $u_{i}+u_{d}$ vanishes inside $C$, so that

$$
u_{d}(\mathbf{x})=-u_{i}(\mathbf{x})=\frac{i}{4} \int_{\partial C} \mathrm{~d} S(\mathbf{y})\left[u_{i}(\mathbf{y}) \partial_{n} V_{0}(\mathbf{y}-\mathbf{x})-V_{0}(\mathbf{y}-\mathbf{x}) \partial_{n} u_{i}(\mathbf{y})\right], \quad \mathbf{x} \in C .
$$

Given that the boundary of $C$ is split up, as for $D$ into segments $\partial C_{m}, m=\overline{1, M}$, we can use (11) 1 , in order to write, for some $\mathbf{x}_{\mathbf{0}}$

$$
V_{0}(\mathbf{y}-\mathbf{x})=V_{0}\left(\mathbf{x}-\mathbf{x}_{0}-\left(\mathbf{y}-\mathbf{x}_{0}\right)\right)=\sum_{n=-\infty}^{\infty} V_{n}^{+}\left(\mathbf{x}-\mathbf{x}_{0}\right) U_{n}^{-}\left(\mathbf{y}-\mathbf{x}_{0}\right)
$$


which holds for $\left|\mathbf{x}-\mathbf{x}_{0}\right|>\left|\mathbf{y}-\mathbf{x}_{0}\right|$. Do this for each of the contours choosing $\mathbf{x}_{0}=\mathbf{x}_{m}$ on each $\partial C_{m}$, , so that

$$
u_{d}(\mathbf{x})=-\frac{i}{4} \sum_{m=1}^{M} \sum_{n=-\infty}^{\infty} V_{n}^{+}\left(\mathbf{x}-\mathbf{x}_{m}\right) \int_{\partial C_{m}} \mathrm{~d} S_{m}\left(u_{i}(\mathbf{y}) \partial_{n} U_{n}^{-}\left(\mathbf{y}-\mathbf{x}_{m}\right)-U_{n}^{-}\left(\mathbf{y}-\mathbf{x}_{m}\right) \partial_{n} u_{i}(\mathbf{y})\right)
$$

where we require $\left|\mathbf{x}-\mathbf{x}_{m}\right|>\left|\mathbf{y}-\mathbf{x}_{m}\right|$ on each contour $\partial C_{m}$ (recall that the integral is being considered for $\mathbf{x} \in C$ ). The minus sign in (22) arises since upon expanding about the point $\mathbf{x}_{m}$, the counter-clockwise orientation with respect to the centre $\mathbf{x}_{m}$ is opposite to the counter-clockwise traversal of $\partial C$ with respect to some origin inside $C$. Note that for this to hold simultaneously for all $m$ the contours $\partial C_{m}$ must be circular arcs as depicted in Figure 3. Therefore we have proved that $C$ is the region with boundary as the closed concave union of the circular arcs defined by $\left\{a_{m}, \phi_{1}^{(m)}, \phi_{2}^{(m)}\right\}$ and denoted as $\partial C_{m}$, see Figure 1. Finally, using the form for $u_{d}$ given in (2c), we find that

$$
b_{m, n}=-\frac{i}{4} \int_{\partial C_{m}} \mathrm{~d} S_{m}\left[u_{i}(\mathbf{y}) \partial_{n} U_{n}^{-}\left(\mathbf{y}-\mathbf{x}_{m}\right)-U_{n}^{-}\left(\mathbf{y}-\mathbf{x}_{m}\right) \partial_{n} u_{i}(\mathbf{y})\right] .
$$

This agrees with [5, Eq. (8)] apart from a factor $i / 4$ missing there. Equation (23) provides

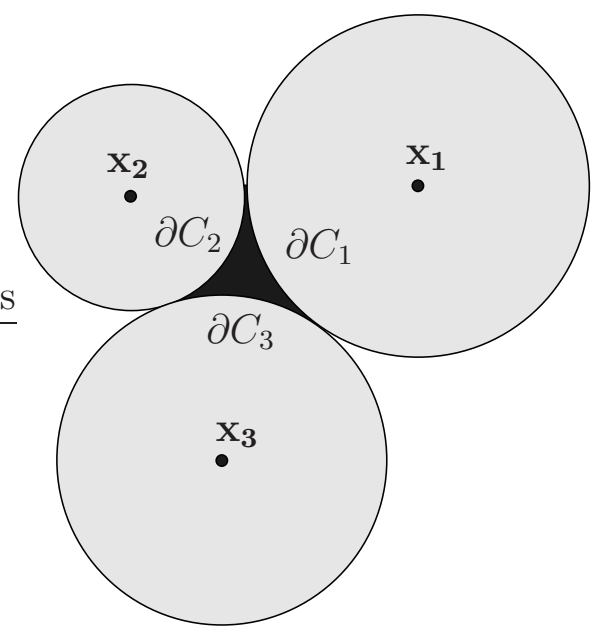

Figure 3: The integration curve $\partial C$ split into $M=3$ portions $\partial C_{m}$ appropriate for the integral representation (22) of the active source field. The cloaked central (black) region, is bounded by $\partial C_{m}, m=1,2,3$.

a direct method for calculating the multipole source amplitudes, as has been demonstrated numerically for different source configurations [5]. The result is not optimal, however, as it requires evaluation of a line integral, which can be computationally time consuming.

The explicit formula for the source amplitudes follows from eq. (23) by introducing the forms for the functions $U_{n}^{-}$as follows

$$
b_{m, l}=-\frac{i}{4} k a_{m} \int_{\phi_{1}^{(m)}}^{\phi_{2}^{(m)}} \mathrm{d} \phi e^{-i l \phi}\left[u_{i}(\mathbf{y}) J_{l}^{\prime}\left(k a_{m}\right)-J_{l}\left(k a_{m}\right) k^{-1} \partial_{n} u_{i}(\mathbf{y})\right] .
$$

We see that the cloaked region $C$ is indeed the subdomain of $D$ in which Graf's theorem can be simultaneously invoked for all of the $M$ active sources. 
Consider plane wave incidence in the direction of the unit vector $\hat{\mathbf{e}}(\psi), u_{i}=u_{\psi}(\mathbf{x})$ where

$$
u_{\psi}(\mathbf{x})=e^{i k \hat{\mathbf{e}}(\psi) \cdot \mathbf{x}} \quad\left(A_{n}=i^{n} e^{-i n \psi)}\right) .
$$

Then (24) becomes, with $\alpha_{m} \equiv k a_{m}$,

$$
\begin{aligned}
b_{m, l} & =\frac{\alpha_{m}}{4 i} u_{\psi}\left(\mathbf{x}_{m}\right) \int_{\phi_{1}^{(m)}}^{\phi_{2}^{(m)}} \mathrm{d} \phi e^{-i l \phi}\left[J_{l}^{\prime}\left(\alpha_{m}\right)-i \mathbf{n}(\phi) \cdot \hat{\mathbf{e}}(\psi) J_{l}\left(\alpha_{m}\right)\right] u_{\psi}\left(\mathbf{y}-\mathbf{x}_{m}\right) \\
& =\frac{\alpha_{m}}{4 i} u_{\psi}\left(\mathbf{x}_{m}\right) \int_{\phi_{1}^{(m)}}^{\phi_{2}^{(m)}} \mathrm{d} \phi\left[J_{l}^{\prime}\left(\alpha_{m}\right)-i \cos (\phi-\psi) J_{l}\left(\alpha_{m}\right)\right] e^{i\left[\alpha_{j} \cos (\phi-\psi)-l \phi\right]} \\
& =\frac{\alpha_{m}}{4 i} u_{\psi}\left(\mathbf{x}_{m}\right) e^{-i l \psi}\left[J_{l}^{\prime}\left(\alpha_{m}\right) G\left(\alpha_{m}\right)-J_{l}\left(\alpha_{m}\right) G^{\prime}\left(\alpha_{m}\right)\right],
\end{aligned}
$$

where the function $G$ is defined as

$$
G(\alpha)=\int_{\phi_{1}^{(m)}-\psi}^{\phi_{2}^{(m)}-\psi} \mathrm{d} \phi e^{i(\alpha \cos \phi-l \phi)}=\sum_{n=-\infty}^{\infty} J_{n}(\alpha) i^{n} \int_{\phi_{1}^{(m)}-\psi}^{\phi_{2}^{(m)}-\psi} \mathrm{d} \phi e^{-i(n+l) \phi} .
$$

The identity $e^{i x \sin \theta}=\sum_{n=-\infty}^{\infty} J_{n}(x) e^{i n \theta}$ has been used in simplifying the form of $G(\alpha)$. Performing the integration in (27), we arrive at an explicit expression for the amplitude coefficients

$$
b_{m, l}=u_{\psi}\left(\mathbf{x}_{m}\right) \frac{\alpha_{m}}{4} \sum_{p=-\infty}^{\infty}\left[J_{p}\left(\alpha_{m}\right) J_{l}^{\prime}\left(\alpha_{m}\right)-J_{p}^{\prime}\left(\alpha_{m}\right) J_{l}\left(\alpha_{m}\right)\right] \frac{i^{p} e^{i p \psi}}{p+l}\left[e^{-i(p+l) \phi_{2}^{(m)}}-e^{-i(p+l) \phi_{1}^{(m)}}\right] .
$$

Now consider the incident field

$$
\frac{i^{-n}}{2 \pi} \int_{0}^{2 \pi} \mathrm{d} \psi u_{\psi}(\mathbf{x}) e^{i n \psi}=U_{n}^{+}(\mathbf{x}) \quad\left(A_{p}=\delta_{n p}\right) .
$$

It follows from integration of (28) that the general form of the amplitude coefficients for the general incidence $(2 \mathrm{~b})$ is given by $(7 \mathrm{~b})$.

Finally, we turn to the question of where the active source field vanishes, noting that the integral (20) vanishes identically for field positions outside $C$ [14]

$$
\frac{i}{4} \int_{\partial C} \mathrm{~d} S(\mathbf{y})\left[u_{i}(\mathbf{y}) \partial_{n} V_{0}(\mathbf{y}-\mathbf{x})-V_{0}(\mathbf{y}-\mathbf{x}) \partial_{n} u_{i}(\mathbf{y})\right]=0, \quad \mathbf{x} \in \mathbb{R}^{2} / C .
$$

How does this relate to the source field $u_{d}(\mathbf{x})$ ? In the course of the derivation of the coefficients $b_{m, n}$ the field $u_{d}(\mathbf{x})$ was expressed in the form (22) for $\mathbf{x} \in C$. The latter restriction on $\mathbf{x}$ can be removed since it is clear that eq. (22) defines $u_{d}(\mathbf{x})$ for all $\mathbf{x}$. This is evident from the definition (2C) and from the identity (23) for $b_{m, n}$. Equation (301) therefore implies that $u_{d}(\mathbf{x})$ vanishes at all positions outside the cloaked region for which the representation (22) holds, i.e. $\left\{\mathbf{x} \notin C:\left|\mathbf{x}-\mathbf{x}_{m}\right|>\left|\mathbf{y}-\mathbf{x}_{m}\right|, \mathbf{y} \in \partial C_{m}, m=\overline{1, M}\right\}$. This is precisely the region $R$ defined in (8), equal to, for instance, the exterior to the colored regions in Figure 3.

This completes the proof of Theorem 2 . 


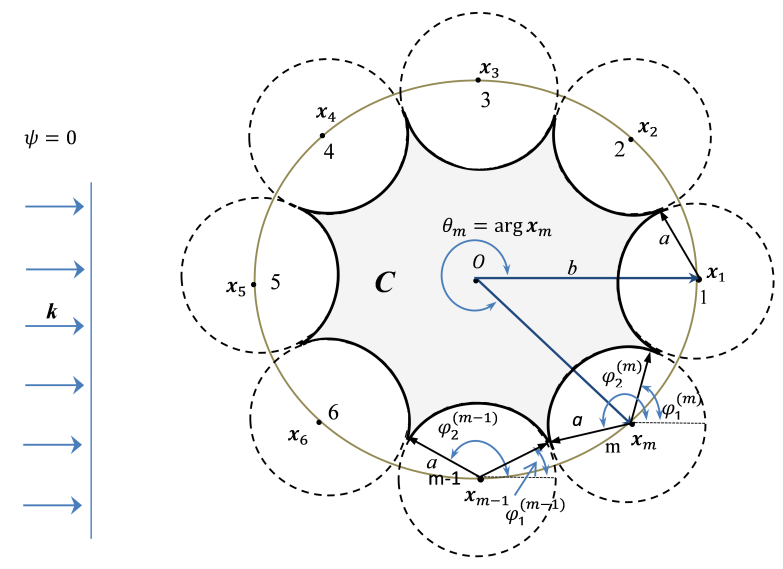

Figure 4: Plane wave insonification of the cloaking region $C$ generated by $M=8$ active sources.

\section{Numerical examples}

\subsection{Active source configuration}

We illustrate the results for plane wave incidence on configurations of the type shown in Fig. 4. The $M$ sources are symmetrically located on a circle, with

$$
a_{m}=a, \quad\left|\mathbf{x}_{m}\right|=b, \quad \theta_{m}=(m-1) \theta_{0} \quad m=\overline{1, M}, \quad \text { where } \theta_{0}=2 \pi / M,
$$

and by necessity, $a \geq b \sin \frac{\pi}{M}$. The circular arcs, which all have the same angular extent, are then defined by

$$
\phi_{1,2}^{(m)}=\pi+\theta_{m} \mp\left|\sin ^{-1}\left(\frac{b}{a} \sin \frac{\pi}{M}\right)-\frac{\pi}{M}\right|, \quad m=\overline{1, M} .
$$

We take $a=b \sin \frac{\pi}{M}$ in all examples considered. Note that the cloaked region $C$ can be formed by a minimum of 3 sources. A configuration with $M=8$ sources is shown in Figure 4. All calculations were performed for plane wave incidence on configurations of the type shown in Figure 4 with varying numbers of sources, $M \geq 3$.

\subsection{Near and farfield amplitudes}

The efficiency of the cloaked region is assessed by examining the farfield and nearfield as functions of various parameters. If all terms in the infinite sums in eqs. (13) and (18) are available then the farfield is identically zero and the nearfield exactly cancels the incident wave, by Theorems 1 and 2. We therefore consider truncated versions of the infinite sums so that the farfield and nearfield coefficients, $F_{n}$ and $E_{n}$ of eqs. (13) and (18) respectively, are approximated as

$$
\left.\begin{array}{l}
F_{n}^{(a p p)} \\
E_{n}^{(a p p)}
\end{array}\right\}=\sum_{m=1}^{M} \sum_{l=-N}^{N} b_{m, l} \times\left\{\begin{array}{l}
V_{n-l}^{-}\left(\mathbf{x}_{m}\right), \\
U_{n-l}^{-}\left(\mathbf{x}_{m}\right),
\end{array} \quad \forall n \in \mathbb{Z} .\right.
$$




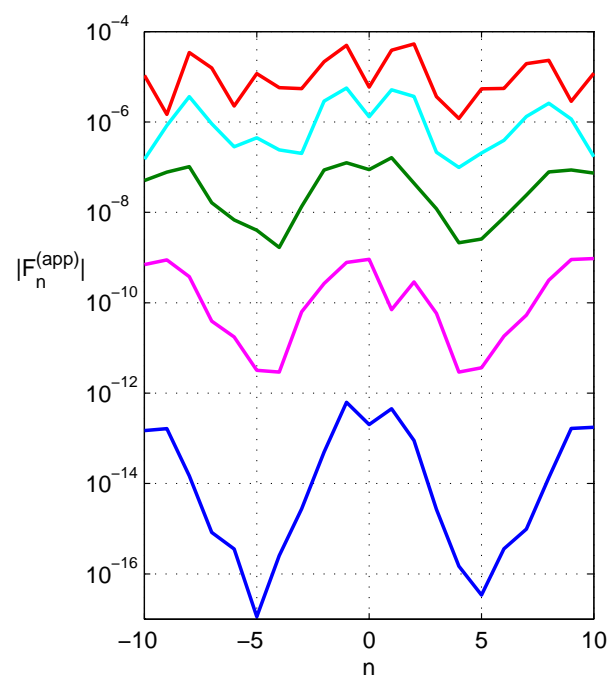

(a) Farfield, $\mathrm{M}=3, \mathrm{~N}=10$

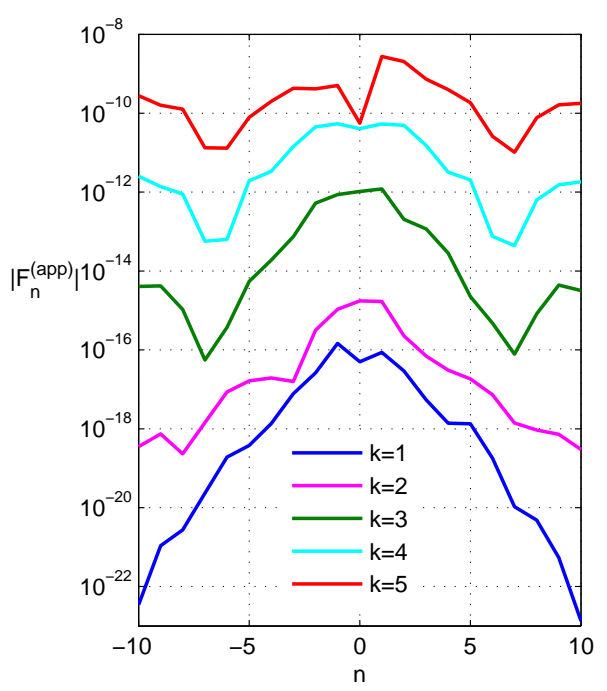

(b) Farfield, $\mathrm{M}=3, \mathrm{~N}=15$

Figure 5: The farfield radiation amplitudes $\left|F_{n}^{(a p p)}\right|$ of eq. (32) for different orders of Bessel functions $n=\overline{-10,10}$, wavenumbers $k=\overline{1,5}$ and truncation values $N=10$ and 15 . The configuration is $M=3$ multipole sources located at the distance $b=1$ from the origin, with angle of incidence $\psi=7^{\circ}$.

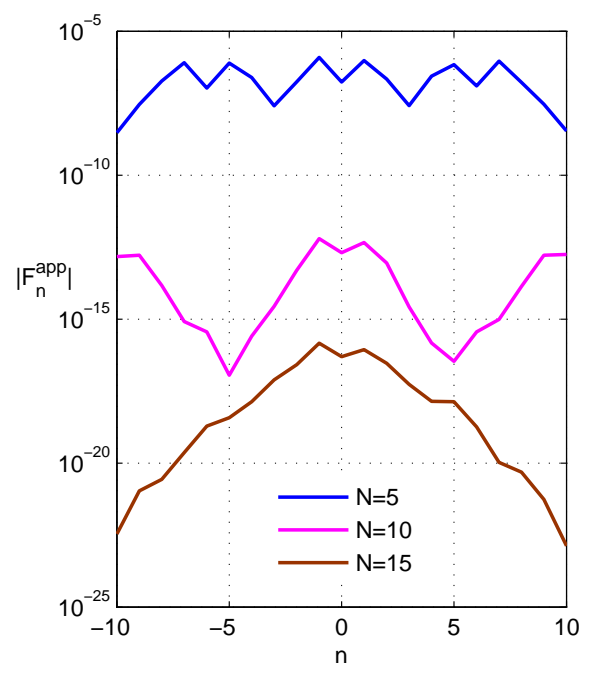

(a) Farfield, $M=3, k=1$

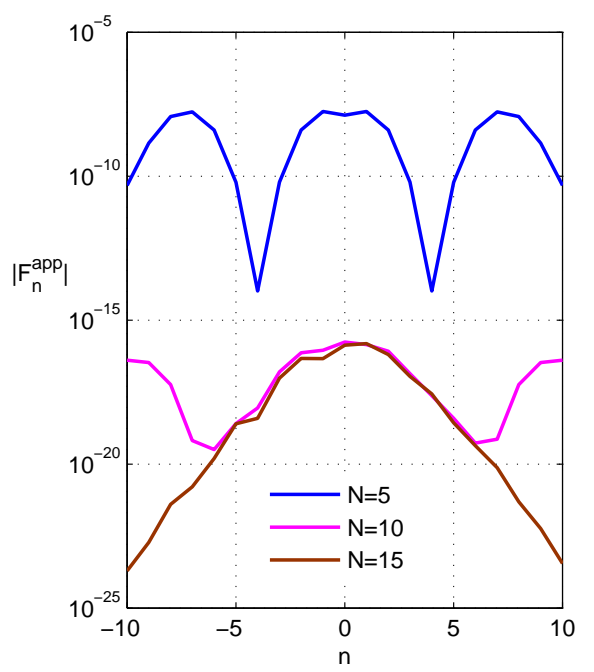

(b) Farfield, $M=8, k=1$

Figure 6: Dependence of the farfield amplitudes $\left|F_{n}^{(a p p)}\right|$ on the order $n$ of Bessel functions for different values of $N$ in (33) $(N=5,10,15)$ and for different numbers of active sources: (a) $M=3$, and (b) $M=8$. The incident wavenumber is $k=1$.

In the limit of $N \rightarrow \infty$ exact cloaking is achieved. Restricting the summation to finite values of $N$ is equivalent to limiting the order of the active multipole sources. The behavior of the approximate coefficients $F_{n}^{(a p p)}$ and $E_{n}^{(a p p)}$ has implications on the accuracy of the cloak regardless of the type of object to be cloaked. Thus, the farfield coefficients determine the radiated field everywhere outside the cloak, and must necessarily be small regardless of 
whether or not an object is being cloaked. Similarly, the total field in the cloaked region $C$ must be small in order to achieve cloaking. The two conditions correspond to $F_{n}^{(a p p)}$ and $E_{n}^{(a p p)}+A_{n}$ having small values. The examples in this subsection examine the sensitivity of these quantities. The sources are located at $b=1$ with plane waves incident at $\psi=17^{\circ}$.

The farfield amplitude coefficients $\left|F_{n}^{(a p p)}\right|, n=\overline{-10,10}$ are depicted in Figures 5 and 6 for different values of the wavenumber $k$, the number of sources $M$, and the number of terms in summation (33), $N$. It is clear from these two figures that the error in the farfield coefficients decreases (i) as $N$ increases, (ii) as $M$ increases, and (iii) as $k$ decreases. The convergence is particularly fast as a function of $N$. For instance, at $k=1$ the farfield coefficients are uniformly less than $10^{-6}$ for all $M \geq 3$ if $N \geq 5$. Much smaller values $\left(10^{-15}\right.$ or less) for $\left|F_{n}^{(a p p)}\right|$ are easily achieved for moderate values of $N$, e.g. $N=10$.

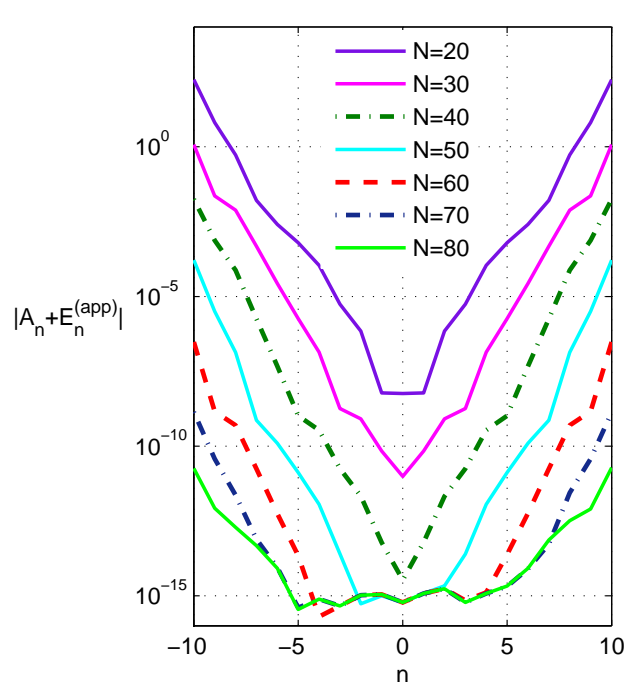

(a) Nearfield, $M=6, k=5$

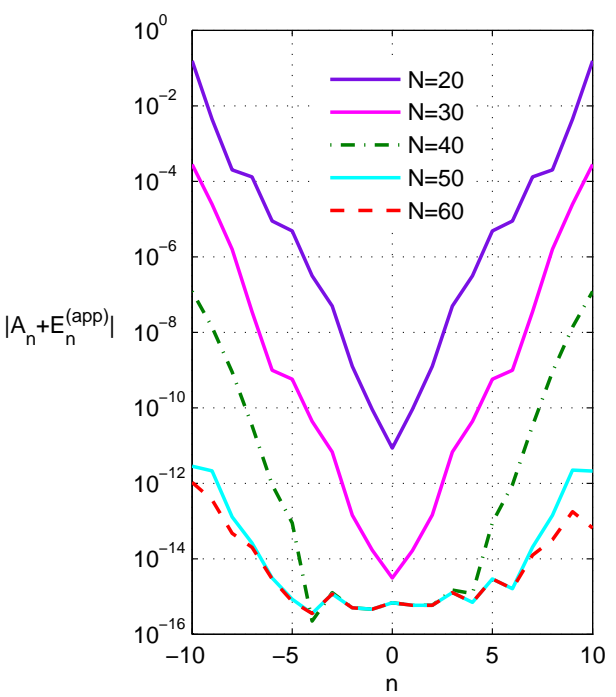

(b) Nearfield, $M=8, k=5$

Figure 7: Variation of the nearfield amplitude coefficients $\left|A_{n}+E_{n}^{(a p p)}\right|$ for different values of the truncation size $N$ in eq. (33), generated by $M=6$ active sources in (a) and $M=8$ sources in (b). In all cases $k=5$.

The nearfield amplitude coefficients $\left|A_{n}+E_{n}^{(a p p)}\right|$ are shown in Figures 7 to 9 , In contrast with the farfield case relatively large values of the truncation size $N$ are required to obtain small nearfield coefficients. Figure 7 shows that $N$ on the order of 100 or more is required to achieve accuracy comparable to the farfield coefficients. However, unlike the farfield amplitudes, it is found that the nearfield coefficients generally increase in magnitude with $|n|$, the order of the Bessel functions. The relatively large values of $\left|A_{n}+E_{n}^{(a p p)}\right|$ and their increase with the order $|n|$ does not necessarily mean that the total field in the nearfield is divergent. For instance, the top curve in Figure $7\left(\right.$ a) indicates $\left|A_{10}+E_{10}^{(a p p)}\right|=\mathrm{O}\left(10^{2}\right)$, but this value multiplies $J_{10}(k r)$, and, for instance, $\left|J_{10}(k r)\right|<2 \times 10^{-3}$ within $C$. In other words, the increasing values of $\left|A_{n}+E_{n}^{(a p p)}\right|$ with $n$ can be balanced by the fact that $J_{n}(k r)=\frac{1}{n !}\left(\frac{k r}{2}\right)^{n}+\ldots$ for small $k r$.

Figure 8 shows the dependence of the nearfield coefficients on the number of sources. The case of the minimum number of sources, $M=3$, appears to be strikingly different from others $(M \geq 4)$. As Figure 8 indicates, adding one more source and taking $M=4$ reduces the error from $10^{0}$ to $10^{-10}$ for $k=1, n= \pm 5$ and from $10^{-2}$ to $10^{-14}$ for $k=5, n= \pm 5$. 


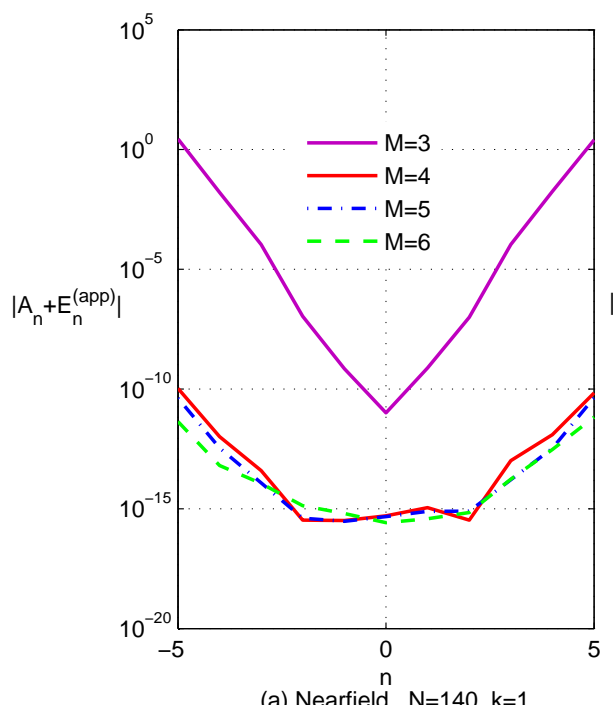

(a) Nearfield, $\mathrm{N}=140, \mathrm{k}=1$

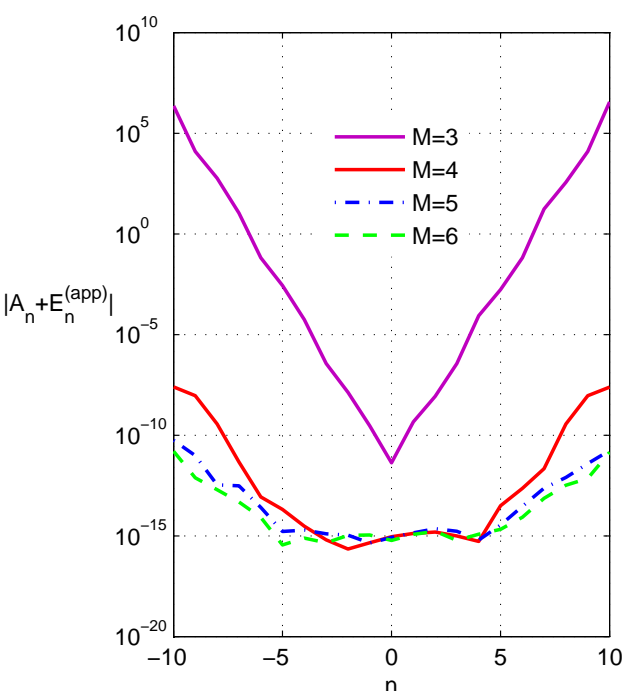

(b) Nearfield, $\mathrm{N}=140, \mathrm{k}=5$

Figure 8: Dependence of the nearfield amplitude $\left|A_{n}+E_{n}^{(a p p)}\right|$ on the number of multipole sources $M(M=3,4,5,6)$ at wavenumber $k=1$ in (a) and $k=5$ in (b).

Generally, as with the farfield coefficients, increasing the number of sources improves the accuracy of the nearfield amplitudes $\left|A_{n}+E_{n}^{(a p p)}\right|$.

Finally, Figure 9 shows the nearfield dependence on the wavenumber, $k=\overline{1,5}$. The accuracy actually improves with increasing $k$, unlike the farfield case. However, it should be borne in mind that the nearfield coefficients multiply the terms $J_{n}(k r)$, which increase in magnitude with $k$ for fixed $r$.
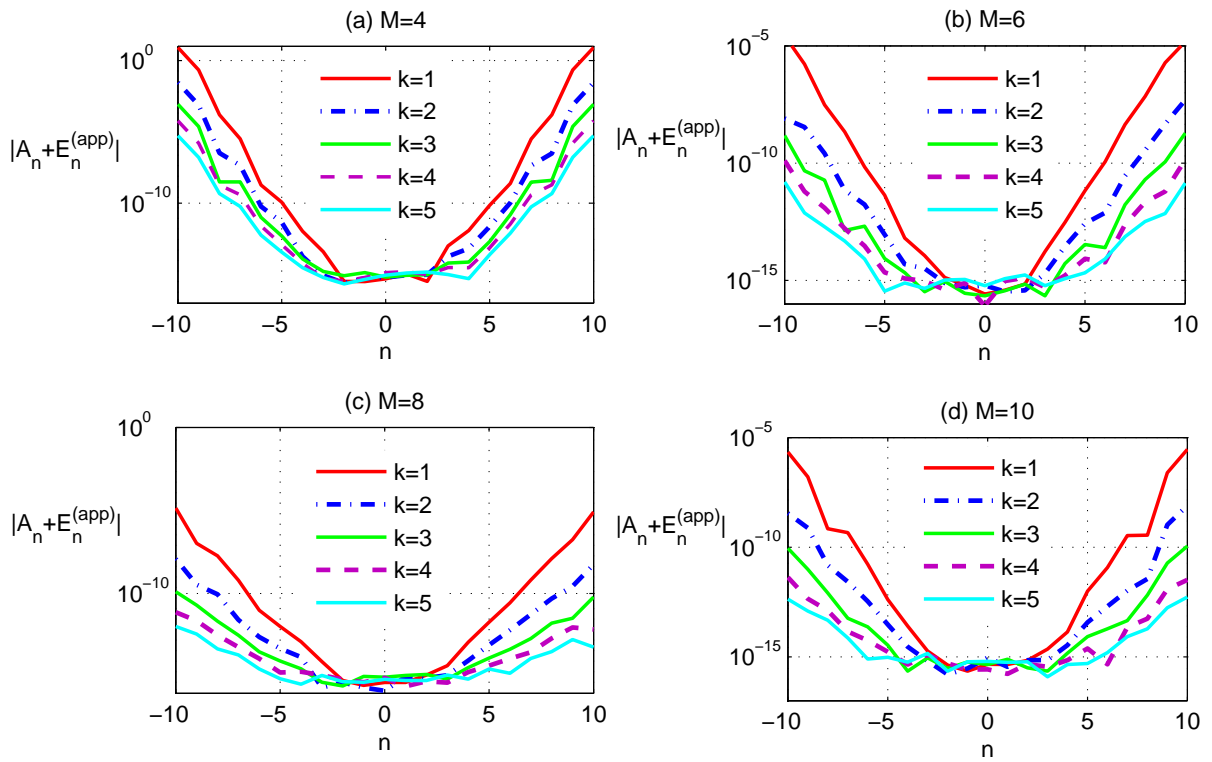

Figure 9: Variation of the nearfield amplitude coefficients with number of active sources $(M=4,6,8,10)$ and with wavenumber $(k=\overline{1,5})$. In all cases $N=130$.

The numerical results in Figures 5 through 9 show that greater accuracy is achieved 
using more sources, which is not unexpected. For the case of $M=3$, the minimum number required, the nearfield coefficients could be large enough to significantly diminish the cloaking effect. This suggests taking $M=4$ might be preferable.
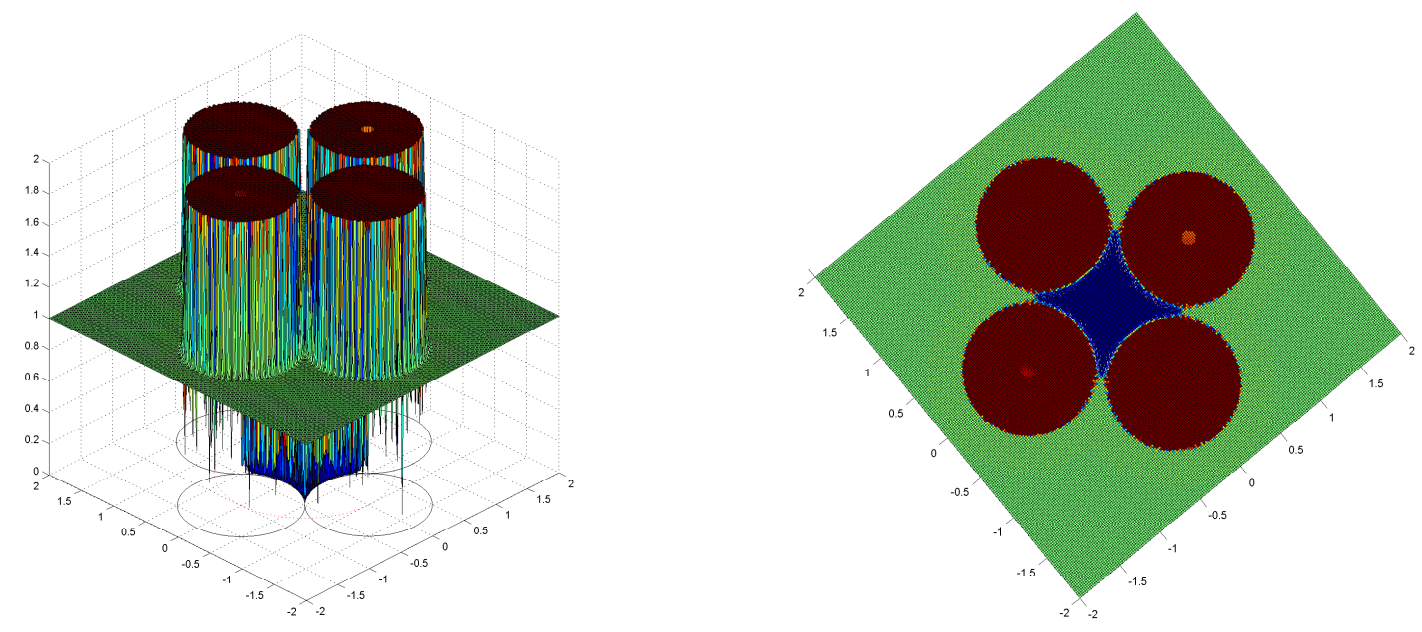

Figure 10: Absolute value of total pressure field with 4 active sources, $b=1$, angle of incidence $\psi=17^{\circ}$, wave number $k=2$, and $N=60$. Values above 2 in magnitude are clipped to make the plots visible.
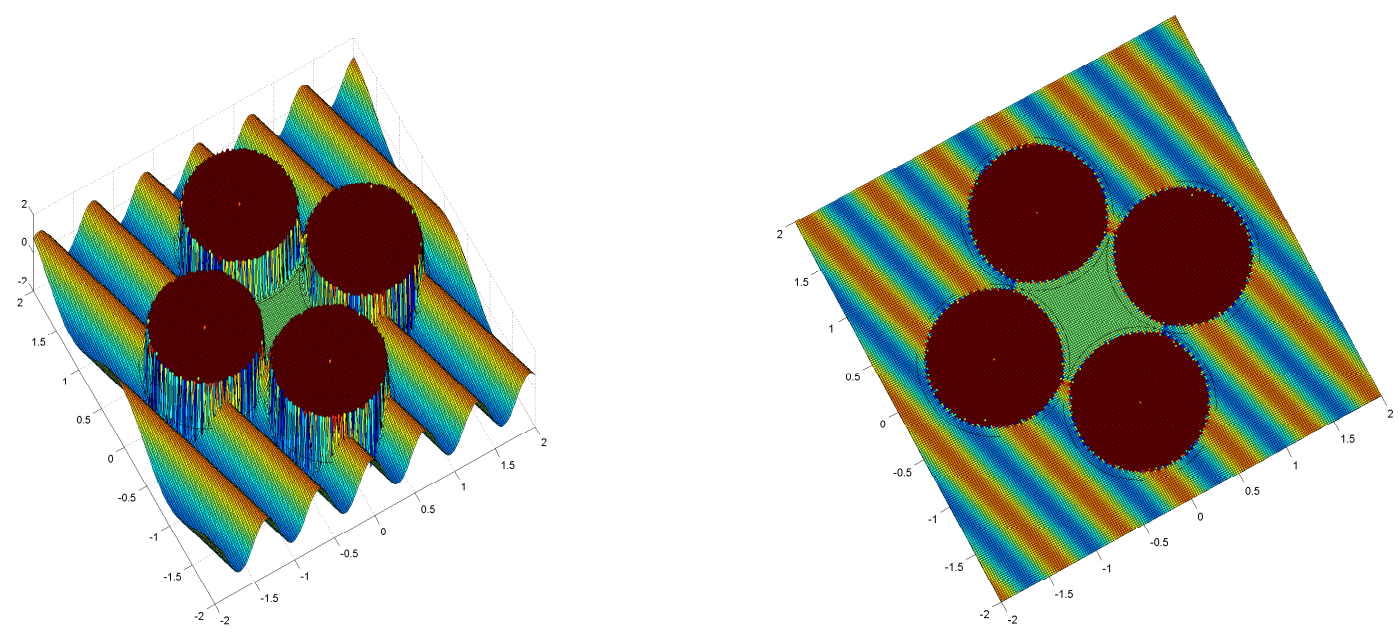

Figure 11: Real part of total pressure field with 4 active sources, $b=1, \psi=17^{\circ}$, wave number $k=10$, and $N=60$. 


\subsection{Total field}

The total field for unit amplitude plane wave incidence on configurations of active sources of the type defined in $\$ 4.1$ is illustrated through several examples. In all cases $b=1$ and $\psi=17^{\circ}$. Figure 10 shows the absolute value of the field for four active sources: the subplots provide different perspectives, indicating that the field is indeed essentially zero in the cloaked region $C$, and that the radiated field $u_{d}$ is zero outside the region $R$. The major variation in the source field is within the circular regions centered on the active sources. It is found that the field in these regions can take very large values, and therefore, for the sake of visibility we truncate the plot at an arbitrary value (here $=2$ ). Note also that the cloaked region spills over slightly into the circular regions. This effect is perhaps easier to see in the subsequent examples.

Figure 11 considers the same $M=4$ configuration of active sources at a higher frequency $k=10$. The plots in this case show the real part of the total field, clearly illustrating the plane wave field in the exterior of $R$. The subplot on the right clearly shows that the cloaked region is somewhat larger than $C$, extending partly into the circular regions. The number of modes used in Figure $11(N=60)$ is more than adequate to ensure convergence and accurate cloaking. It is more instructive to consider the effect of fewer modes, as in Figures 12 to 14. In Figure 12 the number of modes used is on the order of the frequency, and good accuracy is still observed. Notice the smaller footprints of the active sources, as compared with Figure 11, indicating that the higher modes "fill out" the regions where $u_{d}$ is highly variable. Only $N=5$ modes are used in Figure 13, and one can see the deterioration of the cloaking effect expected with an inadequate number of multipoles. The plane wave is clearly evident inside the cloaked region $C$, as is some scattering effects in the "shadow" zone. it is interesting to note that the active source footprints are reduced in size as compared with Figure 12. Finally, in Figure 14, we consider the effect of a larger number of active sources combined with a small number of modes. Comparison of Figures 13 and 14 indicates the tendency observed from the results of $\$ 4.2$ that more active sources improves the cloaking effect. This is also to be expected from the discussion below in $\$ 5$ which shows that for large numbers of sources only the lowest order multipoles play a significant role.
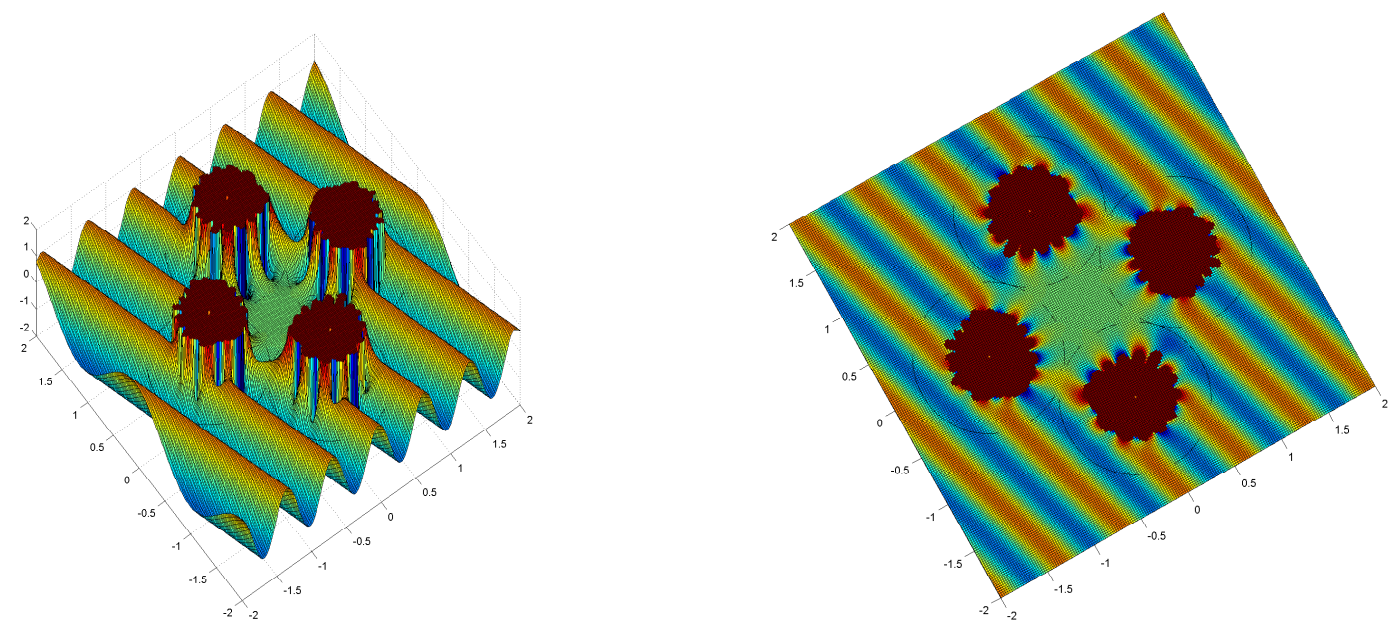

Figure 12: Real part of total pressure field with 4 active sources, $\psi=17^{\circ}, k=10$. The number of modes used in the truncated sum is here limited by $N=10$. 

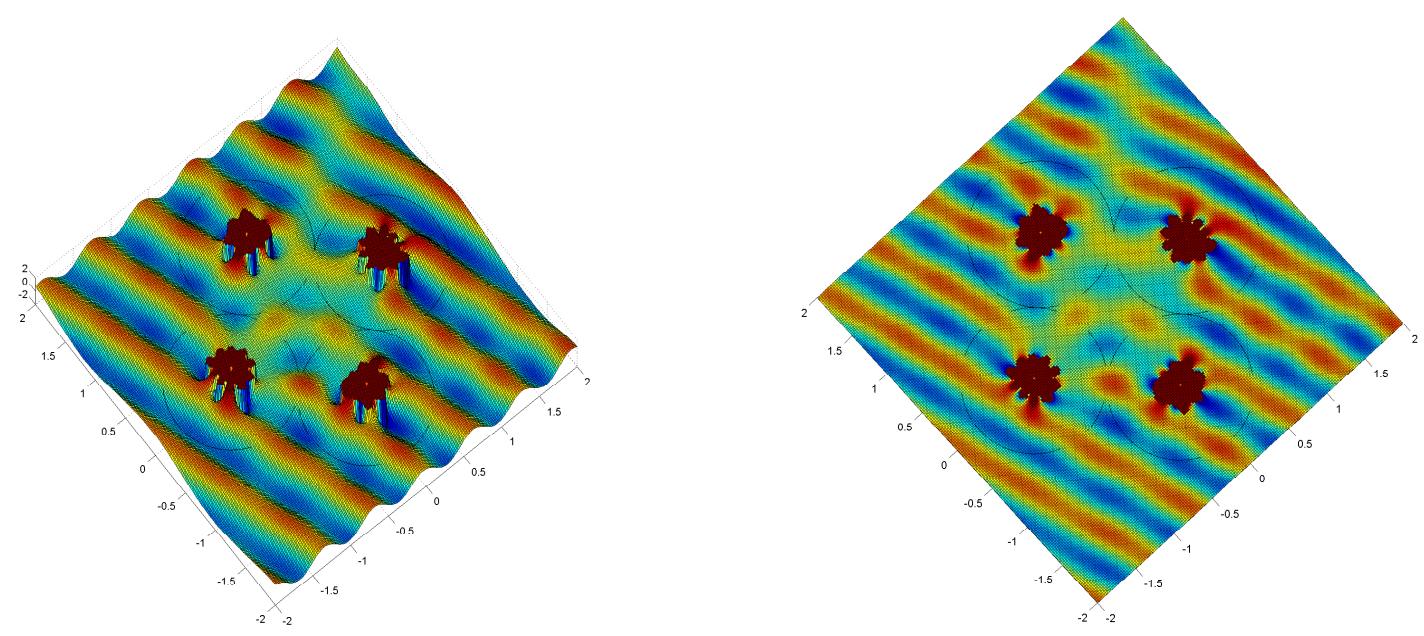

Figure 13: The same as in Figure 12 except now $N=5$.
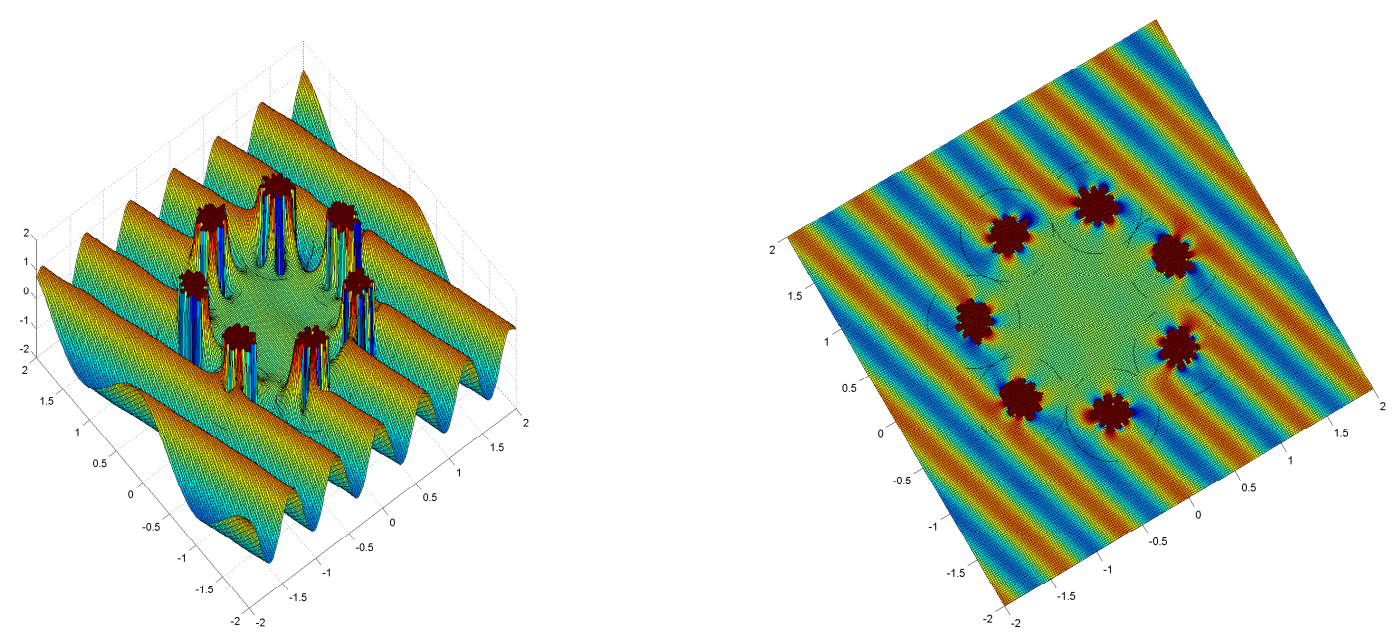

Figure 14: The same as in Figure 13 except now $M=7$.

\subsection{Scattering examples}

Finally, we illustrate the effect of active exterior cloaking on plane wave scattering from rigid and soft cylinders (Neumann and Dirichlet boundary conditions, respectively). In each case the cylinder is circular of radius $a_{0}=1$ centered at the origin, five active sources with $b=4$ are used, the frequency is $k=5$, and the incident wave strikes at angle $\psi=17^{\circ}$.

Figures 15 and 16] compare the response from a rigid cylinder with the active cloaking turned on and turned off. The absolute value is shown in Figure 15 while Figure 16 considers only the real part of the complex field, which clearly indicates the plane wave propagating 
undisturbed when the cloak is active. The comparison for a soft cylinder is shown in Figure 17.
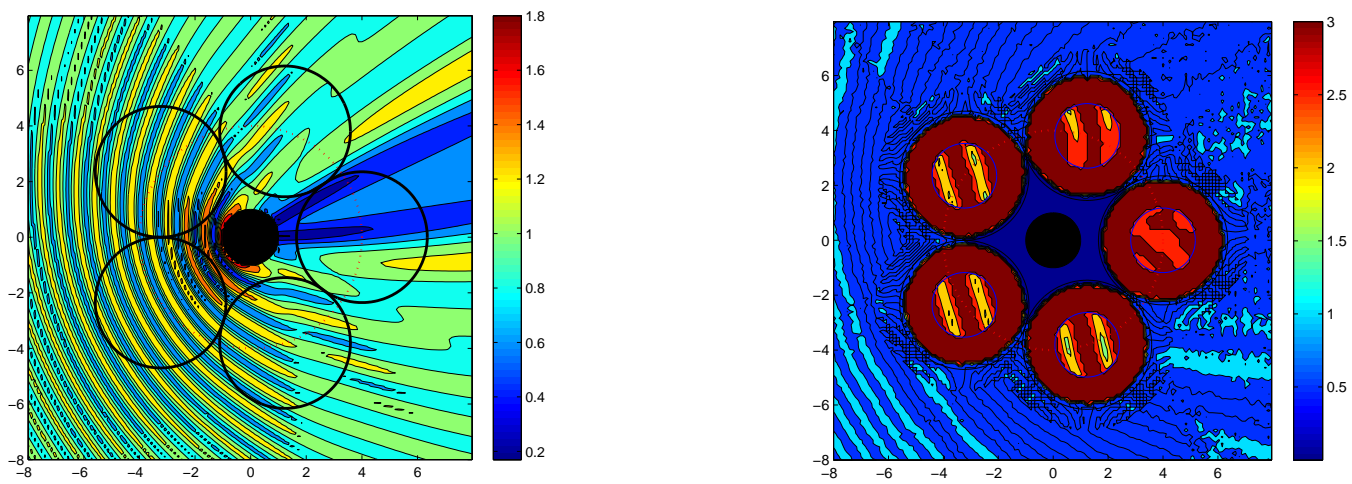

Figure 15: Absolute value of total pressure field when cloaking devices are inactive (left) and active (right) for scattering from the hard cylinder. Calculations are performed for a hard cylinder with $M=5$ active sources, angle of incidence $\psi=17^{\circ}$, and wave number $k a=5$.
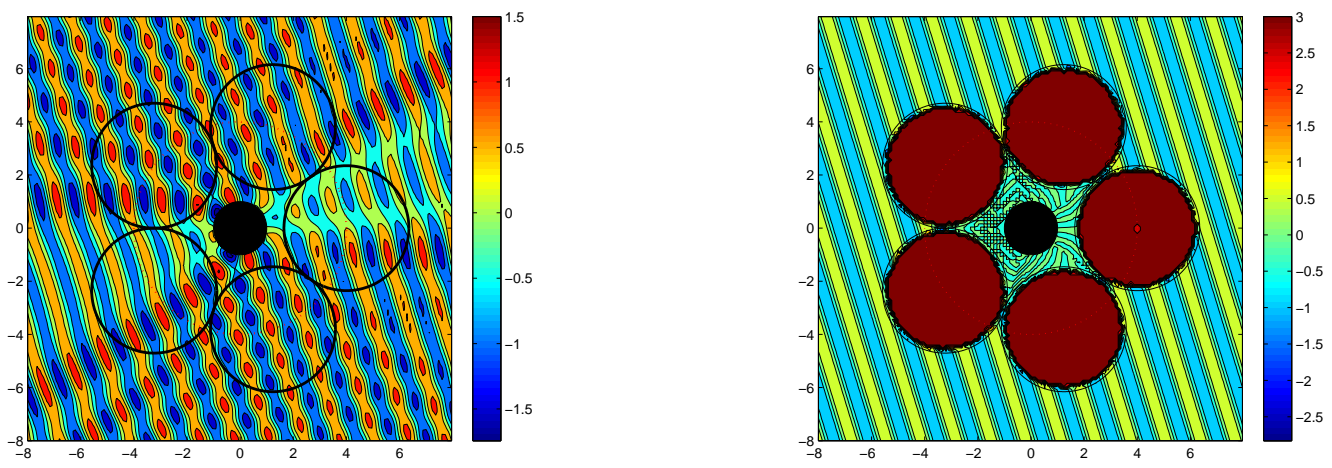

Figure 16: Real value of total pressure field when cloaking devices are inactive (left) and active (right) for scattering from a hard cylinder.

\section{Discussion}

\subsection{The case of many sources: $M \gg 1$}

The numerical results of 44.2 indicate better convergence properties for fewer multipoles if more active sources are used. It is therefore of interest to consider the limit in which many sources are available: the large $M$ limit. Staying with the configuration of 4 .1 the appropriate limit to consider is $\left(a_{m}=\right) a \approx b \pi / M$ so that $k a \ll 1$ and the small argument approximation can be used for the Bessel functions $J_{n}\left(k a_{m}\right)$ (note $k b$ is not necessarily small). 

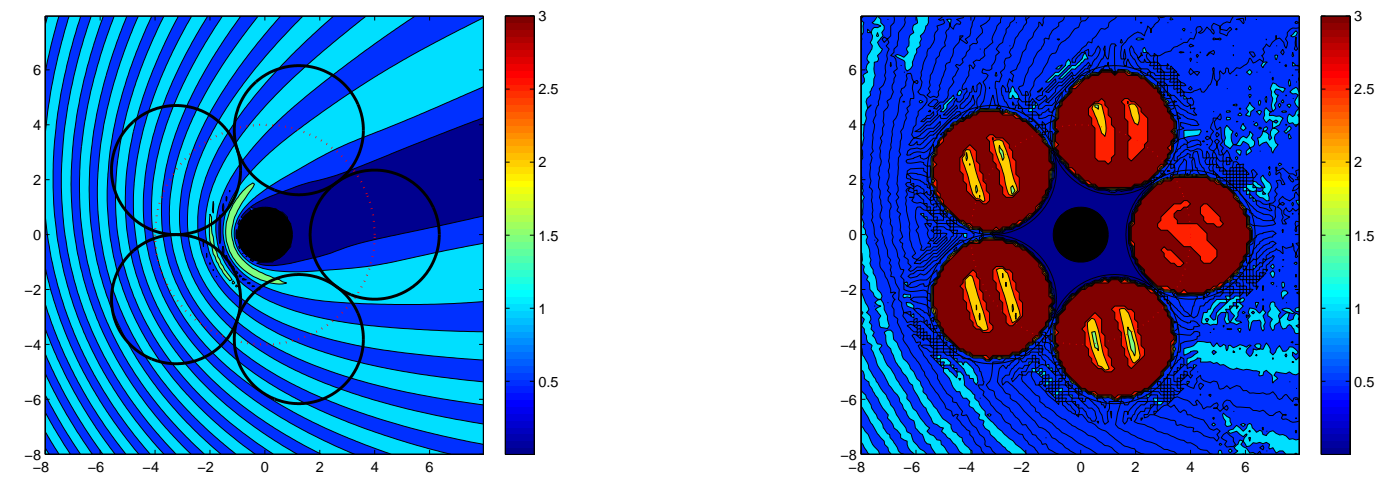

Figure 17: Absolute value of total pressure field with cloaking devices are inactive (left) and active (right) for scattering from a soft cylinder: $k=5, \psi=17^{\circ}$.

This implies that to leading order in $k a$ the coefficients in (7b) reduce to

$$
b_{m, l n}=\frac{i}{4} k a \times \begin{cases}(-2) U_{n}^{+\prime}\left(\mathbf{x}_{m}\right), & l=0 \\ l e^{-i l \theta_{m}} U_{n}^{+}\left(\mathbf{x}_{m}\right), & l= \pm 1 \\ 0, & l \neq 0, \pm 1\end{cases}
$$

The identity $J_{n-1}(x)-J_{n+1}(x)=2 J_{n}^{\prime}(x)$ has been used to simplify the $l=0$ term in (34). The source field follows from eq. (2c) and the identity $V_{-1}^{+}(\mathbf{x})=-V_{1}^{-}\left(\mathbf{x}_{m}\right)$ as

$$
\begin{aligned}
u_{d}= & \frac{i}{2} k a \sum_{n=-\infty}^{\infty} A_{n} \sum_{m=1}^{M} \times \\
& {\left[U_{n}^{+}\left(\mathbf{x}_{m}\right) H_{1}^{(1)}\left(\left|\mathbf{x}-\mathbf{x}_{m}\right|\right) \cos \left(\arg \left(\mathbf{x}-\mathbf{x}_{m}\right)-\theta_{m}\right)-U_{n}^{+^{\prime}}\left(\mathbf{x}_{m}\right) H_{0}^{(1)}\left(\left|\mathbf{x}-\mathbf{x}_{m}\right|\right)\right] . }
\end{aligned}
$$

The field of the active sources is therefore composed of monopoles and dipoles only, with no contribution from higher multipoles. This agrees with what one might expect from the continuous limit of $M \rightarrow \infty$, i.e. a closed contour of monopoles and dipoles, but here it is obtained from the discrete solution. In fact, eq. (35) is

$$
u_{d}=\frac{i}{2} a \sum_{m=1}^{M}\left[u_{i}\left(\mathbf{x}_{m}\right) \partial_{n} V_{0}\left(\mathbf{x}-\mathbf{x}_{m}\right)-V_{0}\left(\mathbf{x}-\mathbf{x}_{m}\right) \partial_{n} u_{i}\left(\mathbf{x}_{m}\right)\right]
$$

which can be seen to be the discretized version of the fundamental integral identity eq. (20) with the correspondence $\int \mathrm{d} S \rightarrow 2 a \sum_{m}$. While eq. (34) is thus the natural first approximation for $u_{d}$ based on the integral equation (20), it should be realized that it was obtained here as a first order approximation of the exact expression (7b). The latter therefore provides the basis for a multipole expansion of the exact source field obtained by including higher powers of $k a$ than considered in eq. (34). This possibility goes beyond our present interests but will be examined in a separate study dealing with approximations to the exact results of Theorem 2 , 


\subsection{Zero scattering matrix}

The exact source field $u_{d}(\mathbf{x})$ of eq. (2c) exhibits some interesting features. This field is, by design, equal to the negative of the incident field in the cloaking region $C$, and it also vanishes identically outside the concave region $R$ defined in (8) The non-radiating property of $u_{d}$ is as important as the fact that it exactly cancels the incident wave in $C$. Let us examine this more closely. Define the infinite matrix $\mathbf{S}$ with elements $S_{p q}$ such that

$$
F_{p}=\sum_{q=-\infty}^{\infty} S_{p q} A_{q} \Rightarrow S_{p q}=\sum_{m=1}^{M} \sum_{l=-\infty}^{\infty} b_{m, l q} U_{p-l}^{-}\left(\mathbf{x}_{m}\right)
$$

or, using (9),

$$
S_{p q}=\left.\sum_{m=1}^{M} \frac{k a_{m}}{4} \sum_{l, n=-\infty}^{\infty} U_{p-l}^{-}\left(\mathbf{x}_{m}\right) U_{n+q}^{+}\left(\mathbf{x}_{m}\right) \frac{(-1)^{n}}{l+n}\left[U_{n}^{-}(\mathbf{a}) U_{l}^{-\prime}(\mathbf{a})-U_{n}^{-\prime}(\mathbf{a}) U_{l}^{-}(\mathbf{a})\right]\right|_{\mathbf{a}_{1}^{(m)}} ^{\mathbf{a}_{2}^{(m)}}
$$

The matrix $\mathbf{S}$ is, formally at least, like a scattering matrix. For instance, by inspection, $\mathbf{S}$ is hermitian $\left(S_{p q}=S_{q p}^{*}\right)$. However, by design and based on Theorem 1, $\mathbf{S} \equiv 0$, and as such it could be called a zero-scattering matrix. Alternatively, it can be viewed as a formula for generating non-radiating fields. This has relevance to the inverse source problem [10]. It is known that solutions to the inverse source problem are non-unique [12, although some uniqueness results are available for restricted forms of sources, e.g. "minimum energy sources" [11]. The solution of the active cloaking problem as developed here has generated a new family of non-radiating sources, with the property that they cancel a given incident field over a finite region.

\section{Conclusions}

By definition, an active source cloaking strategy requires solution of an inverse problem: find the active source amplitudes associated with a given incident field in order to exactly cancel the latter in some finite region. The results given in Theorem 2 provide closed-form solutions for the inverse problem for an arbitrary time harmonic incident wave field. These new expressions require only the expansion of the incident field into entire cylindrical waves and can be evaluated to any degree of accuracy by increasing the truncation parameter $N$ associated with the number of modes of the active source. Simultaneously the fact that the active source field has been shown to vanish identically outside the region $R$ defined in (8) means that the active field is non-radiating. This latter property is just as important as its ability to nullify the incident wave in the region $C$.

The necessary and sufficient conditions on the active source coefficients, given in Theorem 1 provide a means to quantify the error in active cloaking when the number of modes is finite. These errors have been analyzed here in some specific scenarios. It has been shown that the error in the far-field amplitude decreases as $N$ increases, $M$ increases and $k$ decreases. In particular there is a great sensitivity to the increase in $N$; relatively small errors can be attained in the far-field amplitudes for moderate $N$, say $N \sim 10$. On the other hand for small errors in the near-field amplitudes, relatively large values of $N$ are required. Furthermore, there is a striking reduction in error when moving from the case of $M=3$ to $M=4$ motivating the latter as a preference. In contrast to the far-field case, errors decrease for increasing $k$. 
Numerical results were given which illustrate the cloaking effect in various instances, including the presence of a sound-soft and sound-hard circular cylinder. In the appropriate limits, perfect theoretical active cloaking is achieved. The availability of closed-form active source amplitudes opens the door for possible studies on practical realization of active cloaking devices.

The case of many sources, where the active field degenerates to one involving a sum of monopole and dipole sources is worthy of further, separate study relating to the multipole expansion associated with the active field. Finally, the non-radiating nature of the active source field is especially noteworthy. The associated scattering matrix, defined in (38) (which is zero by design) is therefore associated with a new family of non-radiating source solutions which would appear to be useful in the so-called inverse source problem.

\section{References}

[1] F. G. Vasquez, G. W. Milton, and D. Onofrei. Active exterior cloaking for the 2D Laplace and Helmholtz equations. Phys. Rev. Lett., 103:073901, 2009.

[2] D. A. Miller. On perfect cloaking. Opt. Express, 14(25):12457-12466, December 2006.

[3] F. G. Vasquez, G. W. Milton, and D. Onofrei. Broadband exterior cloaking. Opt. Express, 17:14800-14805, 2009.

[4] F. G. Vasquez, G. W. Milton, D. Onofrei, and P. Seppecher. Transformation elastodynamics and active exterior acoustic cloaking. In S. Guenneau and R. Craster, editors, Acoustic Metamaterials: Negative Refraction, Imaging, Lensing and Cloaking, pages 1-1. Canopus Academic Publishing and Springer SBM, 2012.

[5] F. G. Vasquez, G. W. Milton, and D. Onofrei. Exterior cloaking with active sources in two dimensional acoustics. Wave Motion, 49:515-524, 2011.

[6] D. Onofrei and K. Ren. On the active manipulation of quasistatic fields and its applications. arxiv.org/abs/1109.4182, 2011.

[7] F. G. Vasquez, G. W. Milton, and D. Onofrei. Mathematical analysis of the two dimensional active exterior cloaking in the quasistatic regime. arxiv.org/abs/1109.3526, 2011.

[8] G.W. Milton and N.A.P. Nicorovici. On the cloaking effects associated with anomalous localized resonance. Proc. R. Soc. A, 462:30273059, 2006.

[9] H. H. Zheng, J. J. Xiao, Y. Lai, and C. T. Chan. Exterior optical cloaking and illusions by using active sources: A boundary element perspective. Phys. Rev. B, 81:195116+, 2010 .

[10] N. L. Tsitsas and P. A. Martin. Finding a source inside a sphere. Inverse Problems, 28(1):015003+, January 2012.

[11] A. J. Devaney and R. P. Porter. Holography and the inverse source problem. Part II: Inhomogeneous media. J. Opt. Soc. Am. A, 2(11):2006-2011, 1985.

[12] N. Bleistein and J. K. Cohen. Nonuniqueness in the inverse source problem in acoustics and electromagnetics. J. Math. Phys., 18(2):194-201, 1977. 
[13] M. Abramowitz and I. Stegun. Handbook of Mathematical Functions with Formulas, Graphs, and Mathematical Tables. Dover, New York, 1974.

[14] D. L. Colton and R. Kress. Integral Equation Methods in Scattering Theory. Krieger, Melbourne, FL, 1991. 